\title{
Vascular endothelial growth factor expression and pathological changes in the local tissue of facial hemangiomas following injections with pure alcohol
}

\author{
ZHAO-JUN FU ${ }^{1}$, CHUN-MING LI ${ }^{2}$, TAI-HE WANG ${ }^{2}$, ZHU-LING JIANG ${ }^{2}$ and ZHAO-CHEN FU ${ }^{2}$ \\ ${ }^{1}$ Department of Aviation Diseases, General Hospital of Air Force, Beijing 100142; \\ ${ }^{2}$ Department of Oral and Maxillofacial Surgery, The Second Affiliated Hospital of Harbin Medical University, \\ Harbin, Heilongjiang 150001, P.R. China
}

Received March 10, 2014; Accepted July 23, 2014

DOI: $10.3892 / \mathrm{ol} .2014 .2802$

\begin{abstract}
The aim of the present study was to investigate the association between the formation of hemangioma and the expression of vascular endothelial growth factor (VEGF) following local injections of pure alcohol in patients exhibiting hemangioma. Ten healthy subjects (control group) and 10 hemangioma patients (treatment group) were included in the study population, with the hemangioma patients receiving one injection of pure alcohol. The VEGF levels were evaluated in the treatment and control group subjects prior to and following the injection using enzyme-linked immunosorbent assay; furthermore, local tissue was excised to perform pathological analysis one week after the injections. The VEGF levels of the healthy group were identified to be significantly lower when compared with those of the treatment group prior to the injections $(\mathrm{P}<0.01)$ and one week after the injections $(\mathrm{P}<0.01)$, however, were not significantly different when compared with the treatment group one month after the injections $(\mathrm{P}>0.01)$. Therefore, serum VEGF concentrations in the peripheral blood may be a clinical indicator of the efficacy of clinical treatment and aid with determination of the prognosis.
\end{abstract}

\section{Introduction}

The causal factors for hemangiomas have remained unclear until recently. However, it is widely hypothesized that vascular endothelial growth factor (VEGF) is a major factor in the formation of hemangiomas. Shima et al (1) proposed that a shortage of oxygen initially prompts an organism to produce

Correspondence to: Dr Zhao-Chen Fu, Department of Oral and Maxillofacial Surgery, The Second Affiliated Hospital of Harbin Medical University, 246 Xuefu Road, Harbin, Heilongjiang 150001, P.R. China

E-mail: fuzhaochen@163.com

Key words: hemangioma, vascular endothelial growth factor, pure alcohol, enzyme-linked immunosorbent assay
VEGF (1), and VEGF is a type of vessel growth factor, which stimulates the formation of hemangiomas and has previously been investigated in detail (2). Its primary biological functions are as follows: i) Selectively enhancing vascular endothelial cell proliferation and promoting the formation of blood vessels; and ii) participating in the mechanism that increases the permeability of blood vessels, particularly microvessels $(3,4)$. By facilitating the ability to exude large molecules of plasma from a vessel into the surrounding tissue, VEGF promotes the growth of tumor cells, accelerates the formation of novel blood vessels and provides nutrition for these novel vessels (5).

There are various treatment methods for hemangiomas, including surgery, laser treatment, cryotherapy, partial radiation therapy and local injections. Local injections of therapeutic agents are administered directly into the blood vessels within the hemangioma. The agents commonly administered in clinical practice include the following: Corticosteroids, $5 \%$ morrhuate sodium, pingyangmycin and pure alcohol, amongst others. Pure alcohol is a traditional Chinese medicinal compound and its primary ingredients are gal, alum, glycerin, chlorobutanol and stabilizer compounds. Pure alcohol damages the cells of the endothelium of a hemangioma, resulting in clumping of the red blood cells, protein coagulation and thrombosis (6). These effects result in thrombus formation in the lumina of hemangiomas. Therefore, the method of injecting pure alcohol is simple, there is less damage to the patient and the range of applications is wide.

Enzyme-linked immunosorbent assay (ELISA) was used to determine the concentrations of serum VEGF in 10 healthy subjects and 10 hemangioma patients. The 10 hemangioma patients were injected with pure alcohol one week prior to the collection of blood samples, and eight of the hemangioma patients were subsequently injected with pure alcohol one month later. The association between VEGF and the morbidity resulting from hemangiomas was examined. Furthermore, the clinical efficacy of the method of injecting pure alcohol into hemangiomas was observed. In addition, the clinical efficacy of all of the treatment methods for hemangiomas that are adopted at the Department of Oral and Maxillofacial Surgery, the Second Affiliated Hospital of Harbin Medican University (Harbin, China), including laser therapy and surgical removal, 
Table I. Optical density of patient blood samples measured at a wavelength of $450 \mathrm{~nm}$.

Optical density

Group

Patient 1 Patient 2 Patient 3 Patient 4 Patient 5 Patient 6 Patient 7 Patient 8 Patient 9 Patient 10

\begin{tabular}{|c|c|c|c|c|c|c|c|c|c|c|}
\hline Healthy subjects & 0.048 & 0.057 & 0.043 & 0.046 & 0.048 & 0.034 & 0.031 & 0.052 & 0.045 & 0.044 \\
\hline Hemangioma patients & 0.337 & 0.424 & 0.445 & 0.365 & 0.441 & 0.420 & 0.351 & 0.414 & 0.410 & 0.400 \\
\hline \multicolumn{11}{|c|}{ Post pure alcohol injection } \\
\hline 1 week & 0.415 & 0.455 & 0.445 & 0.363 & 0.350 & 0.420 & 0.425 & 0.401 & 0.415 & 0.450 \\
\hline 1 month & 0.058 & 0.051 & 0.050 & 0.042 & 0.045 & 0.059 & 0.060 & 0.054 & & \\
\hline Control & 0.0 & & & & & & & & & \\
\hline
\end{tabular}

are provided based on the experiments that are presented in the current study.

\section{Materials and methods}

Experimental principles. The present study employed the double antibody sandwich ELISA method (Shenzhen Jingmei Biological Engineering Co., Ltd., Shenzhen, China). A rat anti-human VEGF monoclonal antibody (Shenzhen Jingmei Biological Engineering Co., Ltd.) was coated onto an ELISA plate, and standard and biotinylated detection antibody, double antibody, and specimen and standard VEGF samples were added, which formed an 'antibody-VEGF-antibody' compound. The ingredients that did not form part of the compound were washed off. Horseradish peroxidase-labeled avidin and biotin were added to achieve avidin-specific binding. Again, the ingredients that did not combine were washed off and the color reagents, $\mathrm{H}_{2} \mathrm{O}_{2}$ and 3,3',5,5'-tetramethylbenzidine (Shenzhen Jingmei Biological Engineering Co., Ltd.) were added. If VEGF was present, the reagent turned blue. A terminating agent was added and the reaction color changed to yellow. The optical density (OD) was measured at $450 \mathrm{~nm}$; the level of VEGF is proportionate to OD450, whereby a high density of VEGF results in a low OD. This enabled the calculation of the VEGF concentration of the samples by constructing a standard curve.

Specimen collection. Ten normal, healthy subjects (five males and five females; mean age, 46.4 years; range, 20-63 years) were selected at random and served as the control group, with 10 individuals with hemangiomas (six males and four females; mean age, 48.8 years) forming the treatment group (pathological classification: Cavernous hemangioma of; the lower lip [n=3], the tongue $[n=1]$, the upper lip $[n=3]$ and of the cheek $[n=3]$ ). However, two of the 10 hemangioma patients did not complete the follow-up treatment. The treatment process was as follows: Pure alcohol was injected into the tumors of the hemangioma patients using 5-ml disposable syringes following routine sterilization. When the color of the tumor turned pale the injection was stopped. Each of the 10 hemangioma patients was injected with pure alcohol once. Blood samples were obtained prior to the injections, as well as one week and one month after the injections. The blood samples were collected from the patients when they were in a fasted state. Peripheral venous blood (4 ml) was extracted (without anticoagulant) into a centrifuge tube, which was frozen at $-112^{\circ} \mathrm{F}$ in a freezer. One week following the injection of absolute alcohol, the samples were stained with hematoxylin and eosin and observed using a microscope (BX53; Olympus Corporation, Tokyo, Japan). Written informed consent was obtained from all patients and this study was approved by the ethics committee of the Second Affiliated Hospital of Harbin Medical University.

Experimental procedure. ELISA was used in the present study and the human VEGF kits were purchased from Shenzhen China Crystal Co. Ltd (Shenzhen, China). The double antibody sandwich ELISA method was used, which included the following steps. The cleaning mixture was diluted with deionized water at a ratio of 1:20. The reference material comprised of the following: $1.0 \mathrm{ml} \mathrm{Standard/sample}$ diluent was placed into $20 \mathrm{ng}$ of the reference material (at a concentration of $20,000 \mathrm{pg} / \mathrm{ml}$ ) using a $1,000-\mu 1$ pipette. The mixture was allowed to dissolve completely for $15 \mathrm{~min}$ at room temperature, then the reference material was confected at the following concentrations: 1,000, 500, $250,125,62.5,31.25$, and $0 \mathrm{pg} / \mathrm{ml}$. To analyze the blood samples, they were removed from the $-112^{\circ} \mathrm{F}$ freezer and they dissolved completely at room temperature. The blood samples were centrifuged (LD5-10B; Beijing Jingli Centrifuge Co., Ltd., Beijing, China) at a speed of $80 \mathrm{x}$ g for $10 \mathrm{~min}$. The 96-well reaction plates (Shenzhen Jingmei Biological Engineering Co., Ltd.) were coated with the blood samples, and $100 \mu \mathrm{l}$ standard or sample serum was then added to the corresponding well using a $100-\mu 1$ micropipette. The plate was incubated at $98.6^{\circ} \mathrm{F}$ for $90 \mathrm{~min}$ and washed four times with prepared washing solution. A total of $200 \mu$ l biotinylated antibody working solution $(20,000 \mathrm{pg} / \mathrm{ml})$ was added to each well and then the the reaction wells were covered with seal adhesive tape, incubated at $98.6^{\circ} \mathrm{F}$ for $60 \mathrm{~min}$, and washed four times with the prepared washing solution. Enzyme conjugate (100 $\mu \mathrm{l})$ was added to the working solution in each well and the reactions were incubated at $98.6^{\circ} \mathrm{F}$ for $30 \mathrm{~min}$, followed by four sequential washes with the prepared washing solution. The color reagent $(100 \mu \mathrm{l})$ was added to the working solution in each well, and the reactions were incubated at $98.6^{\circ} \mathrm{F}$ for $2 \mathrm{~min}$ and maintained in the dark. Stopping solution $(100 \mu \mathrm{l})$ was added to each well and the reaction solution was mixed. The absorbance was then measured using an ELISA analyzer (9606; Perlong Medical Equipment Co., Ltd., Beijing, China) at a wavelength of $450 \mathrm{~nm}$ (completed within $5 \mathrm{~min}$ ) and the results 
Table II. Levels of VEGF (optical density converted to $\mathrm{pg} / \mathrm{ml}$ ).

VEGF level, pg/ml

Group

Patient 1 Patient 2 Patient 3 Patient 4 Patient 5 Patient 6 Patient 7 Patient 8 Patient 9 Patient 10

\begin{tabular}{lrrrrrrrrrr}
\hline Healthy subjects & 97.3 & 115.1 & 86.5 & 92.7 & 95.3 & 68.3 & 63.6 & 103.3 & 90.2 & 87.3 \\
Hemangioma patients & 650.5 & 849.3 & 890.4 & 730.6 & 881.3 & 840.7 & 701.5 & 828.2 & 820.3 & 800.2 \\
$\begin{array}{l}\text { Post pure alcohol injection } \\
\quad\end{array}$ & & & & & & & & & & \\
$\quad \begin{array}{l}\text { 1 week } \\
\quad \text { month }\end{array}$ & 830.7 & 909.5 & 890.4 & 725.4 & 700.9 & 840.8 & 849.7 & 801.9 & 830.8 & 900.9 \\
Control & 115.4 & 102.3 & 100.8 & 83.5 & 89.9 & 118.9 & 120.7 & 108.7 & & \\
\hline
\end{tabular}

VEGF, vascular endothelial growth factor.

were automatically printed and revealed the OD values (Table I). The results were subsequently converted to units of $\mathrm{pg} / \mathrm{ml}$ (Table II), and a bilateral t-test was performed as part of the statistical analysis (Table III).

The treatment evaluation criteria included the following: i) The outcome was considered excellent if the tumor completely disappeared, there was normal skin and mucosa, no dysfunction and the tongue movement test was negative; ii) the outcome was considered valid if the tumor regression was $>25 \%$ (but did not completely disappear), deformity improved, and the posture mobility test was either negative or positive; and iii) the outcome was considered invalid if the tumor regression was $<25 \%$ or no signs of significant improvement were observed prior to and following treatment.

Statistical analysis. Data were expressed as the mean \pm standard deviation. One-way analysis of variance was performed, and the post hoc Student-Newman-Keuls test was used for multiple comparisons. Statistics were calculated using SPSS version 15.0 for Windows (SPSS Inc., Chicago, IL, USA) and $\mathrm{P}<0.05$ was considered to indicate a statistically significant difference.

\section{Results}

Characteristic features of a tongue hemangioma. A tongue hemangioma is demonstrated in Fig. 1. The following characteristics of a tongue hemangioma were observed: Raised mass, purple and soft on palpation. Fig. 2 shows the local pathological changes that occurred one week after an injection of pure alcohol. The local structure was no longer protruding, the mucosal color had returned to normal, the tongue was freely moveable without dysfunction, and the patient's voice was unchanged. Fig. 3 demonstrates the local pathological changes that were obseved by optical microscopy one week after the injection of pure alcohol. The injection of pure alcohol led to the formation of a thrombus-like structure within the lumen of the hemangioma. Certain regions of the lumen of the hemangioma were blocked completely, whereas other regions were only partially blocked. Such structural characteristics of the lumen ensure a local blood supply and avoid the local formation of necrotic tissue. These factors elucidate the reason that pure alcohol is successful in the treatment of hemangiomas.

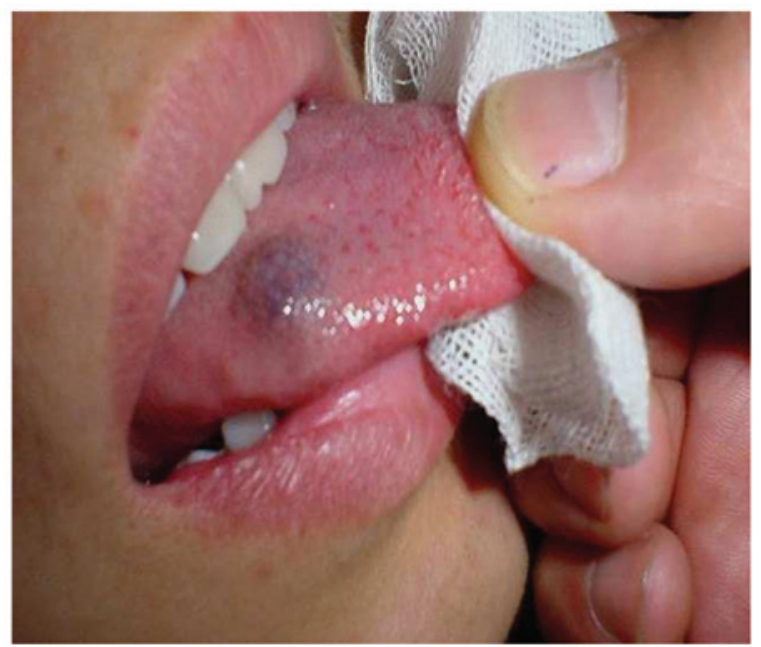

Figure 1. Tongue hemangioma prior to treatment.

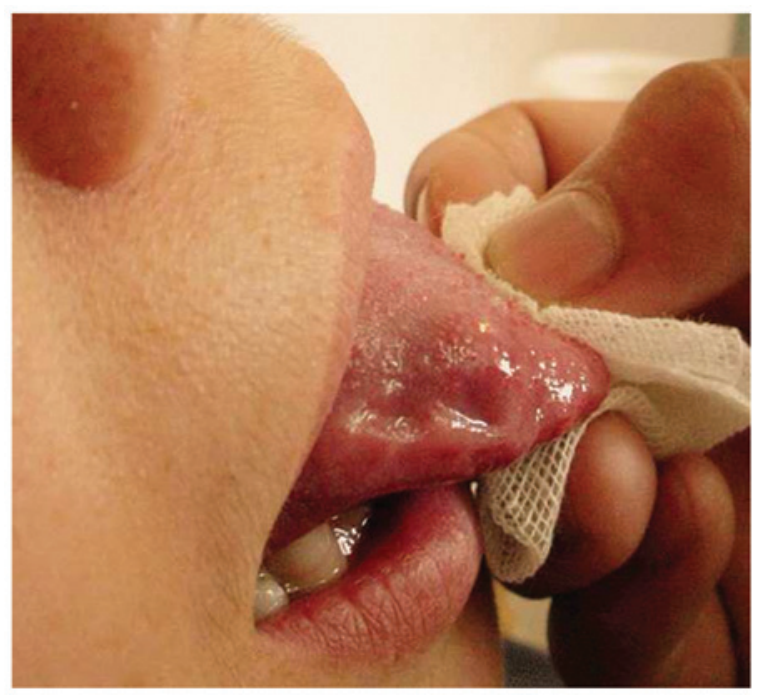

Figure 2. Tongue hemangioma one week following treatment.

Comparison of serum VEGF levels. The serum VEGF levels of the 10 patients with hemangioma were significantly higher when compared with those of the healthy group $(\mathrm{P}<0.01)$. The serum VEGF levels of the 10 hemangioma patients one week 
Table III. VEGF levels in the different groups (presented as the mean \pm standard deviation).

\begin{tabular}{lcc}
\hline Group & Cases, $\mathrm{n}$ & VEGF, pg/ml \\
\hline Healthy subjects & 10 & $97.3 \pm 15.1$ \\
Hemangioma patients & & \\
Prior to injection & 10 & $799.3 \pm 84.1^{\mathrm{a}}$ \\
1 week after injection & 10 & $828.1 \pm 68.8^{\mathrm{a}}$ \\
1 month after injection & 8 & $105.6 \pm 13.5$ \\
\hline
\end{tabular}

${ }^{\mathrm{a}} \mathrm{P}<0.01$ vs. control group. VEGF, vascular endothelial growth factor.

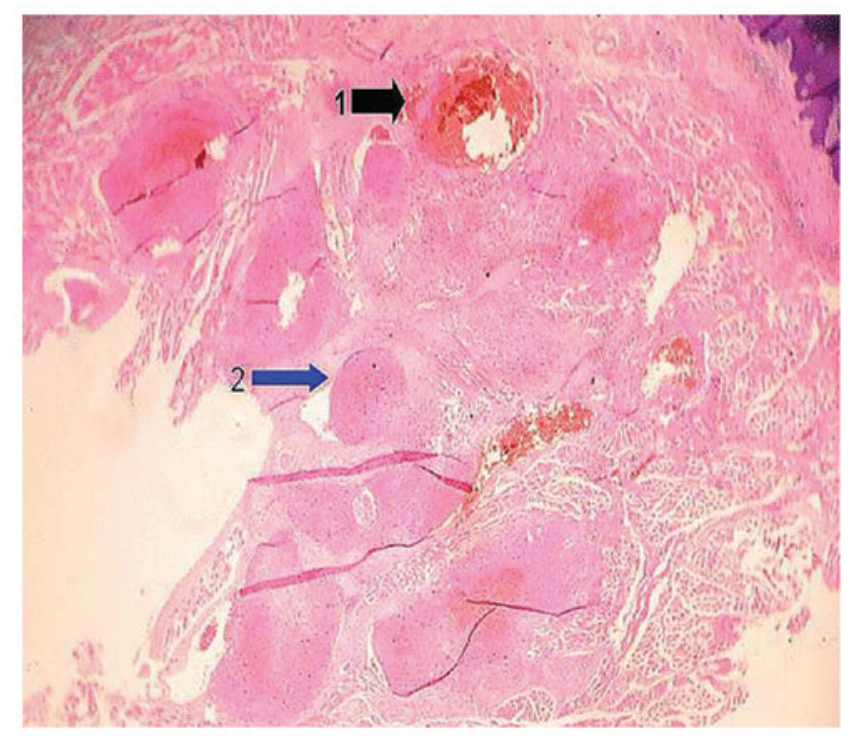

Figure 3. Local pathological changes observed by optical microscopy one week after the injection with pure alcohol. Arrow 1 shows a normal vessel in the hemangioma and arrow 2 shows fibrosis of the local tissue. (stain, hematoxylin and eosin; magnification, x10).

after the pure alcohol injections were not significantly different when compared with the values obtained prior to treatment $(\mathrm{P}<0.01)$, however, were significantly different from the healthy group. This result was due to VEGF secretion by vascular endothelial cells and also by hematopoietic stem cells, such as platelets, megakaryocytes, monocytes and lymphocytes (7). The increase in the VEGF concentration of the peripheral blood was due to the stimulation of local oxidants, which resulted in the synthesis and secretion of compensatory VEGF (8). The serum VEGF levels of eight of the hemangioma patients one week after the pure alcohol injections were not identified to be significantly different than those of the healthy group. The serum VEGF levels in the peripheral blood were close to normal levels. This result was due to the disappearance of the body's inflammatory response one month after the pure alcohol injections, which had metabolized the VEGF (9).

\section{Discussion}

Hemangiomas are a type of congenital benign tumor or vascular malformation with the highest incidence rate observed in infants and young children (10). Hemangiomas, particularly facial lesions, may result in significant psychological distress for the parents as well as the patients (11). Hemangiomas occasionally disappear with age; however, even in cases of complete regression, $38 \%$ of patients continue to present with residual alterations, such as telangiectasias, yellowish discoloration, scarring or dermal atrophy (12). A subset of hemangiomas require active treatment, with the aim of therapy being the reduction or eradication of the hemangioma while minimizing infection, pain and scarring. Numerous treatment modalities have been reported in the literature, ranging from pharmacological therapies to electroacupuncture (13). Treatment options have expanded to include systemic and intralesional corticosteroids, laser ablation, open submucosal resection, and tracheostomy, with a concomitant reduction in the mortality rate observed of $4 \%$ (14).

There are numerous methods for the treatment of hemangiomas, such as surgery, laser treatment, cryotherapy, interferon therapy, hormone therapy, partial radiation therapy, and local injections, as well as others. Pure alcohol injection therapy is frequently used in clinical applications. Currently, there is no objective clinical indicator to assess the therapeutic effects and prognoses following the application of these various treatment strategies. Thus, the aim of the present study was to establish an objective indicator to quantify the therapeutic effect. The exact pathogenesis of hemangiomas remains unclear. The majority of studies hypothesize that VEGF is a direct stimulus for hemangiomas and that the continued elevated expression of it is a key factor in the development of hemangiomas $(15,16)$. VEGF was characterized by Senger et al (17) in 1986 and was initially termed the vascular permeability factor (18). In humans, the VEGF-A gene contains a coding region of $\sim 14 \mathrm{~kb}$ is on chromosome $6 \mathrm{p} 12$. The gene has eight exons that are interspersed with seven introns. Five distinct VEGF protein isoforms have been identified; VEGF121, VEGF145, VEGF165, VEGF189 and VEGF206. Although these VEGF proteins are structurally similar, they are characterized by variations in function and differences in binding specificity. There are important environmental effects of VEGF expression, for example, hypoxia is the most potent agonist of VEGF induction in vitro and in vivo (19). The expression of VEGF mRNA is induced rapidly and reversibly by hypoxia in numerous cell types, including normal, transformed and tumorigenic cells $(20,21)$. Furthermore, VEGF is a dipolymer composed of two identical subunits (connected via disulfide bonds), with a molecular weight of 17,000-22,000 Da. Depending on alternative splicing during RNA transcription, VEGF can be divided into five subtypes: VEGF121, VEGF145, VEGF165, VEGF189 and VEGF208 (9). There are six VEGF variants, each with similar proteins that are involved in the regulation and differentiation of the vascular system, particularly in the blood and lymph vessels (22). VEGF exerts its effect via VEGF receptors that initially undergo autophosphorylation and subsequently activate phosphatidylcholine-specific phospholipase C (PLC-r). PLC-r hydrolyzes phosphatidylinositol diphosphate, producing diacylglycerol (DAG) and inositol triphosphate. DAG activates protein kinase $\mathrm{C}$, which is present in the cytoplasm, and binds it to the membrane, inducing endothelial cell growth and increasing vascular permeability.

In the present study, the VEGF levels in the hemangioma patient group were significantly higher than those in 
the healthy group $(\mathrm{P}<0.01)$. This finding indicates that the serum concentrations of VEGF increased in the hemangioma patients. The serum VEGF levels of the 10 hemangioma patients one week after the pure alcohol injections were not identified to be significantly different when compared with the levels observed prior to treatment, however, were significantly different from the healthy group. This result occurred as VEGF is expressed by vascular endothelial cells, and by hematopoietic stem cells, such as platelets, megakaryocyte, monocytes and lymphocytes (23). However, VEGF is also expressed by endothelial cells, macrophages, and activated smooth muscle cells in atherosclerotic lesions and is expressed as a result of in-stent restenosis neovascularization $(8,24,25)$. The increase in VEGF concentration in the peripheral blood one week after the injections was due to the stimulation of local oxidants, resulting in the synthesis and secretion of compensatory VEGF. The serum VEGF levels in the peripheral blood had almost returned to baseline (similar to the levels of the control subjects) one month after the pure alcohol injections. This result occurred due to the disappearance of the body's inflammatory response one month after the pure alcohol injections, resulting in metabolization of excess levels of VEGF.

In conclusion, the clinical effects of local pure alcohol injections into hemangiomas in this study were consistent with previous studies. The ELISA measurements of the serum VEGF concentration in the peripheral blood accurately reflected the concentrations of VEGF in the blood. Therefore, the serum VEGF concentration in the peripheral blood may be used as a clinical indicator of the efficacy of clinical treatment and to determine the prognosis. The present study provides the basis for future scientific research and clinical investigations.

\section{References}

1. Shima DT, Adamis AP, Ferrara N, Yeo KT, Yeo TK, Allende R, et al: Hypoxic induction of endothelial cell growth factors in retinal cells: identification and characterization of vascular endothelial growth factor (VEGF) as the mitogen. Mol Med 1: 182-193, 1995.

2. Greenberger S, Boscolo E, Adini I, Mulliken JB and Bischoff J: Corticosteroid suppression of VEGF-A in infantile hemangioma-derived stem cells. N Engl J Med 362: 1005-1013, 2010.

3. Xiao X, Liu J and Sheng M: Synergistic effect of estrogen and VEGF on the proliferation of hemangioma vascular endothelial cells. J Pediatr Surg 39: 1107-1110, 2004.

4. Kleinman ME, Greives MR, Churgin SS, Blechman KM, Chang EI, Ceradini DJ, et al: Hypoxia-induced mediators of stem/progenitor cell trafficking are increased in children with hemangioma. Arterioscler Thromb Vasc Bio 27: 2664-2670, 2007.

5. Tokuyama W, Mikami T, Masuzawa M and Okayasu I: Autocrine and paracrine roles of VEGF/VEGFR-2 and VEGF-C/VEGFR-3 signaling in angiosarcomas of the scalp and face. Hum Pathol 41: 407-414, 2010.

6. Elmasri H, Karaaslan C, Teper Y, Ghelfi E, Weng M, Ince TA, et al: Fatty acid binding protein 4 is a target of VEGF and a regulator of cell proliferation in endothelial cells. FASEB J 23: 3865-3873, 2009.
7. Haggstrom AN, Drolet BA, Baselga E, Chamlin SL, Garzon MC Horii KA, et al: Prospective study of infantile hemangiomas: clinical characteristics predicting complications and treatment. Pediatrics 118: 882-887, 2006

8. Span PN, Grebenchtchikov N, Geurts-Moespot J, Westphal JR, Lucassen AM and Sweep CG: EORTC Receptor and Biomarker Study Group Report: a sandwich enzyme-linked immunosorbent assay for vascular endothelial growth factor in blood and tumor tissue extracts. Int J Biol Markers 15: 184-191, 2000.

9. Ozawa CR, Banfi A, Glazer NL, et al: Microenviromental VEGF concentration, not total dose, determines a threshold between normal and aberrant angiogenesis. J Clin Invest 113: 516-527, 2004.

10. Mulliken JB and Glowacki J: Hemangiomas and vascular malforations in infants and children: a classification based on endothelial characteristics. Plast Reconstr Surg 69: 412-422, 1982.

11. Tanner JL, Dechert MP and Frieden IJ: Growing up with a facial hemangioma: parent and child coping and adaptation. Pediatrics 101: 446-452, 1998

12. Liu LJ, Xiao C and Zhu X: New advances in treatment of tumour using VEGF and its receptor. Oncology section of foreign medicine science 28: 113-115, 2001.

13. Bruckner AL and Frieden IJ: Hemangiomas of infancy. J Am Acad Dermatol 48: 477-493, 2003

14. Bitar MA, Moukarbel RV and Zalzal GH: Management of congenital subglottic hemangioma: trends and success over the past 17 years. Otolaryngol Head Neck Surg 132: 226-231, 2005.

15. Tischer E, Mitchell R, Hartman T, Silva M, Gospodarowicz D, Fiddes JC and Abraham JA: The human gene for vascular endothelial growth factor. Multiple protein forms are encoded through alternative exon splicing. J Biol Chem 266: 11947-11954, 1991.

16. Ferrara N: Vascular endothelial growth factor: basic science and clinical progress. Endocr Rev 25: 581-611, 2004.

17. Senger DR, Perruzzi CA, Feder J and Dvorak HF: A highly conserved vascular permeability factor secreted by a variety of human and rodent tumor cell lines. Cancer Res 46: 5629-5632, 1986.

18. Zachary I and Gliki G: Signaling transduction mechanisms mediating biological actions of the vascular endothelial growth factor family. Cardiovase Res 49: 568-581, 2001.

19. Kirito K and Kaushansky K: Thrombopoietin stimulates vascular endothelial cell growth factor (VEGF) production in hematopoietic stem cells. Cell Cycle 4: 1729-1731, 2005.

20. Minchenko A, Bauer T, Salceda S and Caro J: Hypoxic stimulation of vascular endothelial growth factor expression in vitro and in vivo. Lab Invest 71: 374-379, 1994.

21. Mahajan D, Miller C, Hirose K, McCullough A and Yerian L: Incidental reduction in the size of liver hemangioma following use of VEGF inhibitor bevacizumab. J Hepatol 49: 867-870, 2008.

22. Berse B, Brown LF, Van de Water L, Dvorak HF and Senger DR: Vascular permeability factor (vascular endothelial growth factor) gene is expressed differentially in normal tissues, macrophages, and tumors. Mol Biol Cell 3: 211-220, 1992.

23. Kastrup J, Jørgensen E, Rück A, Tägil K, Glogar D, Ruzyllo W, et al; Euroinject One Group: Direct intramyocardial plasmid vascular endothelial growth factor-A165 gene therapy in patients with stable severe angina pectoris. A randomized double-blind placebo-controlled study: the Euroinject One trial. J Am Coll Cardiol 45: 982-988, 2005.

24. Couffinhal T, Kearney M, Witzenbichler B, Chen D, Murohara T, Losordo DW, et al: Vascular endothelial growth factor/vascular permeability factor (VEGF/VPF) in normal and atherosclerotic human arteries. Am J Pathol 150: 1673-1685, 1997.

25. Inoue M, Itoh H, Ueda M, Naruko T, Kojima A, Komatsu R, et al: Vascular endothelial growth factor (VEGF) expression in human coronary atherosclerotic lesions: possible pathophysiological significance of VEGF in progression of atherosclerosis. Circulation 98: 2108-2116, 1998 . 